\title{
THE VOLUME OF HYPERBOLIC ALTERNATING LINK COMPLEMENTS
}

\author{
MARC LACKENBY \\ With An APPEndiX By IAN AgOl and Dylan Thurston
}

\section{INTRODUCTION}

A major goal of knot theory is to relate the geometric structure of a knot complement to the knot's more basic topological properties. In this paper, we will do this for hyperbolic alternating knots and links, by showing that the link's most fundamental geometric invariant - its volume - can be estimated directly from its alternating diagram.

A bigon region in a link diagram is a complementary region of the link projection having two crossings in its boundary. A twist is either a connected collection of bigon regions arranged in a row, which is maximal in the sense that it is not part of a longer row of bigons, or a single crossing adjacent to no bigon regions. The twist number $t(D)$ of a diagram $D$ is its number of twists. (See Figure 1.) Recall that a diagram is prime if any simple closed curve in the diagram that intersects the link projection transversely in two points disjoint from the crossings bounds a disc that contains no crossings. Menasco proved [5] that a link with a connected prime alternating diagram, other than the standard diagram of the $(2, n)$-torus link, is hyperbolic. Our main theorem is the following rather surprising result, which asserts that the link complement's hyperbolic volume is, up to a bounded factor, simply the diagram's twist number.

Theorem 1. Let $D$ be a prime alternating diagram of a hyperbolic link $K$ in $S^{3}$. Then

$$
v_{3}(t(D)-2) / 2 \leq \operatorname{Volume}\left(S^{3}-K\right)<v_{3}(16 t(D)-16)
$$

where $v_{3}(\approx 1.01494)$ is the volume of a regular hyperbolic ideal 3 -simplex.

The upper bound on volume actually applies to any diagram of a hyperbolic link, not just an alternating one. The lower bound on volume can be improved to $v_{3}(t(D)-2)$ if, in addition, $D$ is 'twist-reduced'. We will define this term later in the paper and show that any prime alternating link has a twist-reduced prime 
alternating diagram.

Shortly after this paper was distributed, Dylan Thurston and Ian Agol improved the upper bound in Theorem 1 to $10 v_{3}(t(D)-1)$. Moreover, they showed that this new upper bound is asymptotically sharp, in that there is a sequence of links $K_{i}$ with prime alternating diagrams $D_{i}$ such that Volume $\left(K_{i}\right) / 10 v_{3} t\left(D_{i}\right) \rightarrow$ 1 as $i \rightarrow \infty$. Their results are given in an appendix to this paper.

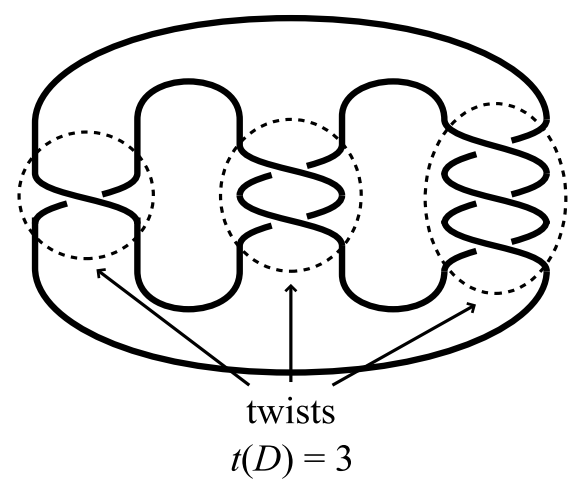

Figure 1.

The following two corollaries are sample applications of Theorem 1. They control convergence of hyperbolic alternating link complements in the geometric topology. We will show that the only limit points are the 'obvious' ones, namely augmented alternating link complements, as defined by Adams in [2].

Corollary 2. A complete finite volume hyperbolic 3-manifold is the limit of a sequence of distinct hyperbolic alternating link complements if and only if it is a hyperbolic augmented alternating link complement.

Corollary 3. The set of all hyperbolic alternating and augmented alternating link complements is a closed subset of the set of all complete finite volume hyperbolic 3-manifolds, in the geometric topology.

The upper bound on volume is proved by using techniques related to the Gromov norm [4]. We will show that the volume of $S^{3}-K$ is at most the volume of a link complement with a diagram having $4 t(D)$ crossings. By constructing an explicit ideal triangulation for this link complement, we find an upper bound for its volume.

The lower bound is established by using a theorem of Agol [3]. When a finite 
volume hyperbolic 3-manifold $M$ contains a properly embedded 2-sided incompressible boundary-incompressible surface $S$, Agol established a lower bound on the volume of $M$ in terms of the 'guts' of $M-\operatorname{int}(\mathcal{N}(S))$. In our case, $M$ is the complement of $K$, and $S$ is the orientable double cover of one of the two 'checkerboard' surfaces arising from an alternating diagram.

\section{The UPPer BOUnd ON VOLUMe}

We will use the fact [8] that if a compact orientable hyperbolic 3-manifold $M$ is obtained by Dehn filling another hyperbolic 3-manifold $N$, then the volume of $M$ is less than the volume of $N$. The 3 -manifold $N$ we will use is the exterior of the link $J$ that is obtained by replacing each twist of the diagram $D$ with a tangle containing at most six crossings. This tangle is composed of the two original strings of the twist, but with all but two (respectively, all but one) of its crossings removed, depending on whether the twist contained an even (respectively, odd) number of crossings. Those two strings are then encircled with a simple closed curve, as in Figure 2, known as a crossing circle. (There is one exception to this: if two of these crossing circles cobound an annulus in the complement of the remaining link components, then only one of these should be used.) The resulting link $J$ is an augmented alternating link, and hence is hyperbolic [2]. The link $K$ is obtained from $J$ by performing $1 / q$ surgeries, for certain integer values of $q$, on the crossing circles. Hence, $\operatorname{Volume}\left(S^{3}-K\right)<\operatorname{Volume}\left(S^{3}-J\right)$. If we alter the diagram of $J$ near each crossing circle by removing the residual crossing(s) of the twist, the result is a new link $L$. By [1], $\operatorname{Volume}\left(S^{3}-L\right)=\operatorname{Volume}\left(S^{3}-J\right)$.

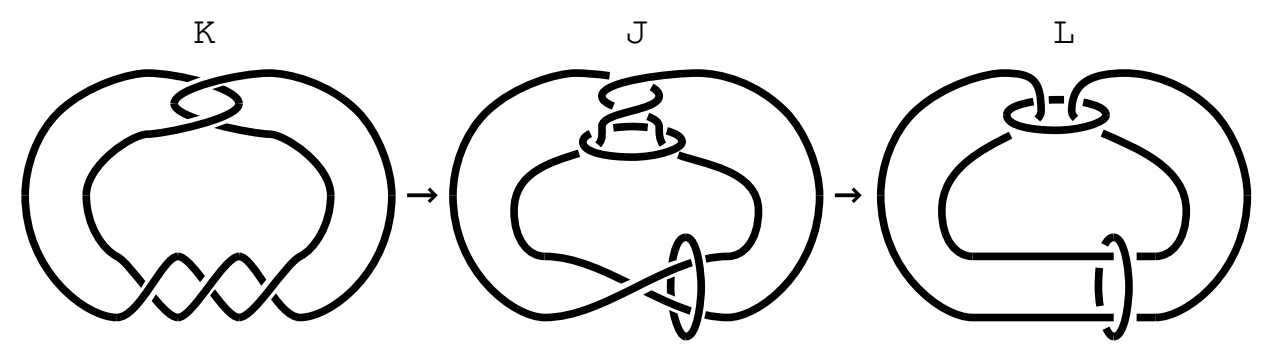

Figure 2 .

Note the diagram $D_{L}$ of $L$ is prime and connected, since $D$ is prime, connected and not the standard diagram of the $(2, n)$-torus link. Hence, it determines a 
decomposition of $S^{3}-L$ into two ideal polyhedra with their faces identified in pairs [4]. Here, we are using the term polyhedron in quite a general sense: a 3-ball with a connected graph in its boundary that contains no loops and no valence one vertices. An ideal polyhedron is a polyhedron with its vertices removed. The edges of this ideal polyhedral decomposition of $S^{3}-L$ are vertical arcs, one at each crossing. The faces are the regions of the diagram, twisted a little near the crossings so that their boundaries run along the link and the edges, and so that the interiors of the faces are disjoint. The remainder of $S^{3}$ is two open 3-balls, which we take to be the interiors of the two ideal polyhedra $P_{1}$ and $P_{2}$.

Note that the intersection of the 2-skeleton with the boundary tori of the link exterior is a 4-valent graph. Using Euler characteristic, the number of complementary regions of this graph is equal to the number of vertices. The former is the number of vertices of the ideal polyhedra. The latter is $2 c\left(D_{L}\right)$, where $c\left(D_{L}\right)$ is the number of crossings in the diagram $D_{L}$, since there are two vertices for each edge of the polyhedral decomposition.

We now subdivide the faces of the polyhedra with more than three boundary edges into triangles by coning from an ideal vertex. We wish to calculate the resulting number of triangles. Let $V, E$ and $F$ be the total number of ideal vertices, edges and faces in the boundary of the two ideal polyhedra. So,

$$
V-E+F=\chi\left(\partial P_{1}\right)+\chi\left(\partial P_{2}\right)=4
$$

The number of triangles is the sum, over all faces of the polyhedra, of the number of sides of the face minus two. This is $2 E-2 F=2 V-8=4 c\left(D_{L}\right)-8$.

Now collapse each bigon face of the polyhedra to a single edge. Some care is required here, since it is a priori possible that there is a cycle of bigons, glued together along their edges. However, an examination of the diagram $D_{L}$ gives that the bigons are in fact disjoint.

In each polyhedron, there is a vertex with valence at least four. Otherwise, the boundary graph is a single triangle or the tetrahedral graph, and it is straightforward to check that these graphs do not arise. For example, observe that each region of $D_{L}$ has an even number of sides and there must be more than two nonbigon regions. So each polyhedron ends up with more than four triangular faces. 
Triangulate each polyhedron by coning from this vertex. The result is an ideal triangulation of the complement of $L$ with at most $4 c\left(D_{L}\right)-16$ tetrahedra.

This allows us to bound the volume of the complement of $L$. We homotope each ideal tetrahedron so that it lifts to a straight simplex in the universal cover $\mathbb{H}^{3}$. First homotope each edge, preserving its ends, so that it is either a geodesic or has been entirely homotoped into a cusp. Then homotope each ideal 2-simplex so that it is straight, but possibly degenerate. Then do the same for the 3 simplices. The volume of each resulting ideal 3 -simplex is at most $v_{3}$. Hence, $\operatorname{Volume}\left(S^{3}-L\right) \leq v_{3}\left(4 c\left(D_{L}\right)-16\right)=v_{3}(16 t(D)-16)$. This proves the righthand inequality of the main theorem. Note again that we did not use that $D$ is alternating.

\section{THE LOWER BOUND ON VOLUME}

The lower bound on the volume of a hyperbolic alternating link is proved using the following theorem of Agol [3]. It deals with a finite volume hyperbolic 3-manifold $M$ containing a properly embedded incompressible boundaryincompressible surface $S$. We denote $M-\operatorname{int}(\mathcal{N}(S))$ by $M_{S}$.

Theorem. (Agol [3]) Let $M$ be an orientable hyperbolic 3-manifold containing a properly embedded orientable boundary-incompressible incompressible surface $S$. Then

$$
\operatorname{Volume}(M) \geq-2 v_{3} \chi\left(\operatorname{Guts}\left(M_{S}\right)\right)
$$

We refer the reader to [3] for a full description of the 'guts' terminology. Essentially, the pair $\left(M_{S}, \partial \mathcal{N}(S) \cap \partial M_{S}\right)$ has an associated characteristic submanifold $\Sigma$, which is a canonical collection of $I$-bundles and Seifert fibred spaces embedded in $M_{S}$, and the guts of $M_{S}$ is the closure of the complement of $\Sigma$. We refer to $P=\partial M \cap \partial M_{S}$ as the parabolic locus. It is a collection of annuli and tori.

Note that the assumption that $S$ is orientable can be dropped, providing the surface $\tilde{S}=\operatorname{cl}(\partial \mathcal{N}(S)-\partial M)$ is incompressible and boundary-incompressible. For

if we apply Agol's theorem to $\tilde{S}$, then $M_{\tilde{S}}$ is a copy of $\mathcal{N}(S)$ and a copy of $M_{S}$. The former is an $I$-bundle and hence a component of the characteristic submanifold of $M_{\tilde{S}}$. Hence, $\operatorname{Guts}\left(M_{\tilde{S}}\right)=\operatorname{Guts}\left(M_{S}\right)$. 
In our case, $M$ is the exterior of the alternating link $K$, and $S$ is one of the two checkerboard surfaces $B$ and $W$, arising from a diagram of $K$. These surfaces arise by colouring the regions of the diagram black and white, so that regions meeting along an arc of the link projection have different colours. If all the faces with the same colour are glued together, twisted near the crossings, the result is one of the checkerboard surfaces. However, instead of using the given diagram $D$ of $K$, it is convenient to work with a diagram that is in addition twist-reduced. This means that whenever a simple closed curve in the diagram intersects the link projection transversely in four points disjoint from the crossings, and two of these points are adjacent to some crossing, and the remaining two points are adjacent to some other crossing, then this curve bounds a subdiagram that consists of a (possibly empty) collection of bigons arranged in a row between these two crossings. An equivalent pictorial definition of a twist-reduced diagram is given in Figure 3.

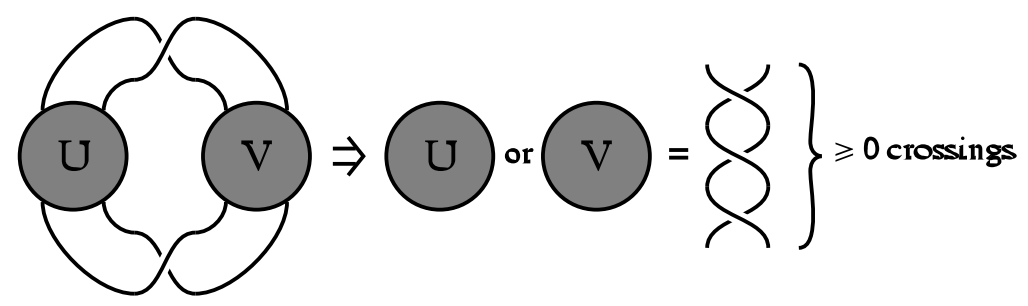

Figure 3.

Any prime alternating link has a twist-reduced prime alternating diagram. For if a diagram is not twist-reduced, it decomposes as in Figure 3, where the top and bottom crossings lie in different twists. There is a sequence of flypes that amalgamates these into a single twist, reducing the twist number of the diagram. However, we need to know more than this. We will prove the following in $\S 4$.

Lemma 4. Let $K$ be a link with a connected prime alternating diagram $D$. Then $K$ has a connected prime alternating twist-reduced diagram $D^{\prime}$ with at least $(t(D) / 2+1)$ twists.

We let $B$ and $W$ be the black and white checkerboard surfaces for the twistreduced diagram $D^{\prime}$. Let $r_{B}\left(D^{\prime}\right)$ and $r_{W}\left(D^{\prime}\right)$ be the number of black and white non-bigon regions of $D^{\prime}$. We will prove the following theorem in $\S 5$. 
Theorem 5. Let $D^{\prime}$ be a prime alternating twist-reduced diagram of $K$, let $M$ be the exterior of $K$, and let $B$ and $W$ be the checkerboard surfaces for $D^{\prime}$. Then

$$
\begin{gathered}
\chi\left(\operatorname{Guts}\left(M_{B}\right)\right)=2-r_{W}\left(D^{\prime}\right) \\
\chi\left(\operatorname{Guts}\left(M_{W}\right)\right)=2-r_{B}\left(D^{\prime}\right) .
\end{gathered}
$$

Note that the diagram $D^{\prime}$ induces a planar graph, with a vertex at each twist and an edge for each edge of $D^{\prime}$ that is not adjacent to a bigon region. Denote the number of its vertices, edges and faces by $V, E$ and $F$. Then $2 E=4 V$, since it is 4 -valent. Hence,

$$
2=V-E+F=-V+F=-t\left(D^{\prime}\right)+r_{B}\left(D^{\prime}\right)+r_{W}\left(D^{\prime}\right) .
$$

The lower bound on volume follows rapidly from these results. Adding the inequalities in Agol's theorem applied to $B$ and $W$, we obtain

$$
\begin{aligned}
\operatorname{Volume}\left(S^{3}-K\right) & \geq-v_{3}\left(\chi\left(\operatorname{Guts}\left(M_{B}\right)\right)+\chi\left(\operatorname{Guts}\left(M_{W}\right)\right)\right) \\
& =-v_{3}\left(4-r_{B}\left(D^{\prime}\right)-r_{W}\left(D^{\prime}\right)\right) \\
& =v_{3}\left(t\left(D^{\prime}\right)-2\right) \\
& \geq v_{3}(t(D) / 2-1) .
\end{aligned}
$$

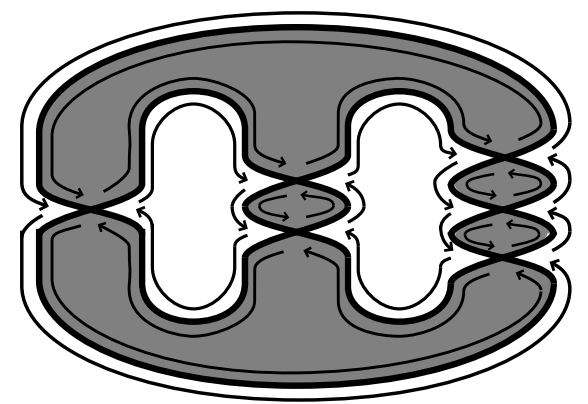

Figure 4.

The proof of Theorem 5 relies heavily on the ideal polyhedral decomposition of a link complement arising from a connected prime diagram, as described in $\S 2$. Its 2-skeleton is the union of the two checkerboard surfaces. When the diagram is alternating, the boundary graphs of the two polyhedra $P_{1}$ and $P_{2}$ are particularly simple. They are just copies of the underlying 4 -valent graph of the link projection 
[4]. Each region of one polyhedron is glued to the corresponding region of the other, with a rotation that notches the face around one slot in either a clockwise or anti-clockwise direction, depending on whether the region is coloured white or black. Thurston compared this gluing procedure to the gears of a machine [8].

Throughout much of this paper we will consider a surface $S$ properly embedded in $M_{B}$ (or $M_{W}$ ). It will intersect the parabolic locus $P=\partial M \cap \partial M_{B}$ in a (possibly empty) collection of transverse arcs. It will be incompressible and also parabolically incompressible (see Figure 5) which means that there is no embedded disc $E$ in $M_{B}$ such that

- $E \cap S$ is a single arc in $\partial E$;

- the remainder of $\partial E$ is an arc in $\partial M_{B}$ which has endpoints disjoint from $P$ and which intersects $P$ in at most one transverse arc;

- $E \cap S$ is not parallel in $S$ to an arc in $\partial S$ that contains at most one component of $\partial S \cap P$.

Also no component of $S$ will be a boundary-parallel disc or a 2-sphere.

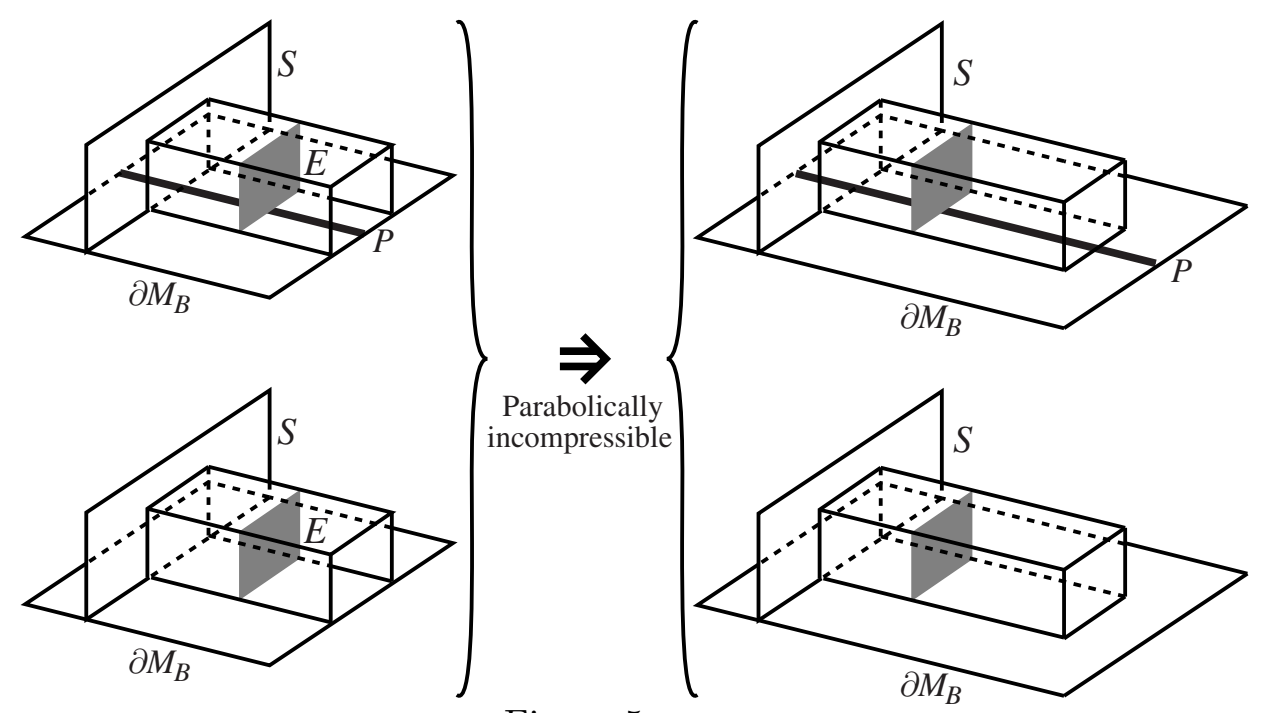

Figure 5.

A fairly standard argument gives that such a surface $S$ can be ambient isotoped (leaving $P$ invariant) into normal form, which means it satisfies the following conditions: 
- the intersection of $S$ with each of the ideal polyhedra $P_{i}$ is a collection of properly embedded discs;

- each such disc intersects any boundary edge of $P_{i}$ at most once;

- the boundary of each such disc cannot enter and leave an ideal vertex through the same face of $P_{i}$;

- $S$ intersects any face of $P_{i}$ in a collection of arcs, rather than simple closed curves;

- no such arc can have endpoints in the same ideal vertex of $P_{i}$, or in a vertex and an edge that are adjacent;

- no component of intersection between $S$ and $\partial P_{i}$ forms the boundary of a regular neighbourhood of an edge.

In fact, for this argument to work, we need to know that $\partial \mathcal{N}(B) \cap M_{B}$ is incompressible and boundary-incompressible in $M_{B}$, but we will check this below. The last of the above conditions is non-standard. It can be guaranteed since $S$ is properly embedded in $M_{B}$ rather than $M$.

Using normal surfaces, we can prove the following lemma, which we need in order to apply Agol's theorem. This is a stronger version of a result of Menasco and Thistlethwaite (Proposition 2.3 of [6]) that asserts that $B$ and $W$ are incompressible in $M$.

Lemma 6. The surfaces $\tilde{B}=\partial \mathcal{N}(B) \cap M_{B}$ and $\tilde{W}=\partial \mathcal{N}(W) \cap M_{W}$ are incompressible and boundary-incompressible in $M$.

Proof. If there is a compression disc for $\tilde{B}$, say, then there is one $S$ in normal form. The intersection of $S$ with $W$ can contain no simple closed curves, since such a curve would lie in a face of the polyhedral decomposition. So it is a collection of arcs. Suppose initially that there is at at least one such arc. An outermost one in $S$ separates off a disc $S^{\prime}$ in $S$ that lies in some $P_{i}$. Its boundary intersects the edges of $P_{i}$ twice and misses the vertices. The boundary graph of $P_{i}$ is a copy of the link diagram. Since the diagram is prime, we deduce that $\partial S^{\prime}$ intersects the same edge of $P_{i}$ twice. This contradicts the definition of normality. Therefore, $S \cap W$ is empty. But, then $S$ lies entirely in one $P_{i}$, with its boundary in a black 
face. It is therefore not a compression disc.

If there is a boundary-compression disc for $\tilde{B}$, then there is one in normal form. As above, we may assume that $S \cap W$ is empty. But $S$ then lies in one $P_{i}$, with $\partial S$ running over a single ideal vertex and avoiding all edges of $P_{i}$. This contradicts the fact that the diagram is prime.

\section{The Characteristic DeComposition of A Link Diagram}

Analogous to the characteristic submanifold of a 3-manifold, in this section we define the characteristic decomposition of a connected prime link diagram $D$. We will consider simple closed curves in the diagram. Always these will be disjoint from the crossings and will intersect the link projection transversely. We will, at various points, isotope these curves. Always, the isotopy will leave the crossings fixed throughout. In this section, we will make this proviso without further mention.

A square is a simple closed curve that intersects the link projection four times. The link projection divides it into four arcs, which we call its sides. A square is essential if it is not homotopically trivial in the complement of the crossings, or, equivalently, it intersects four distinct edges of the link projection. A square is characteristic if it is essential, does not separate off a single crossing, and any other square can be isotoped off it. Taking one isotopy class of each characteristic square, and isotoping them so that they are all disjoint, gives a collection of squares which we term the characteristic collection.

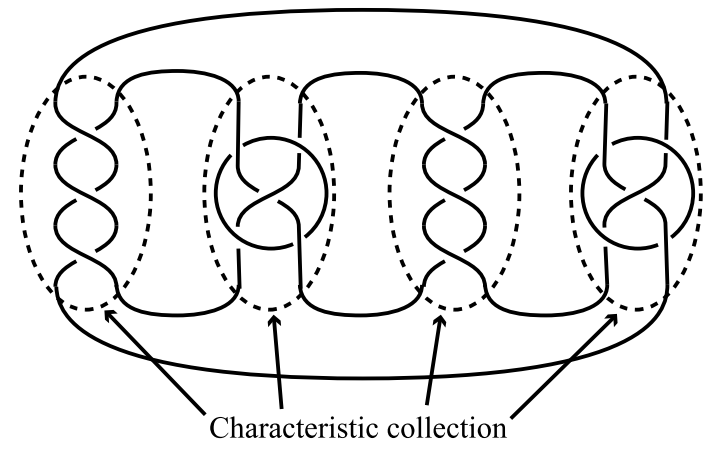

Figure 6 . 
Consider a collection $\mathcal{C}$ of disjoint essential squares. Define a connected complementary region $R$ of $\mathcal{C}$ to be a product region if each component of intersection between $R$ and the black regions (or each component of intersection between $R$ and the white regions) intersects $\mathcal{C}$ and the crossings in a total of two components. (See Figure 7.) Thus, $R$ is a copy of a 2 -sphere with some open discs removed, the open discs and the crossings lying on the equator of the 2-sphere, and the black (or white) regions forming a regular neighbourhood of the remainder of the equator. The reason for the name product region is as follows. If we view this 2 -sphere as the boundary of a 3-ball $B$, then $B$ minus an open regular neighbourhood of the crossings is the product of a closed interval and a disc. The white regions in $R$ form the horizontal boundary of this product, and the black regions lie in the vertical boundary.

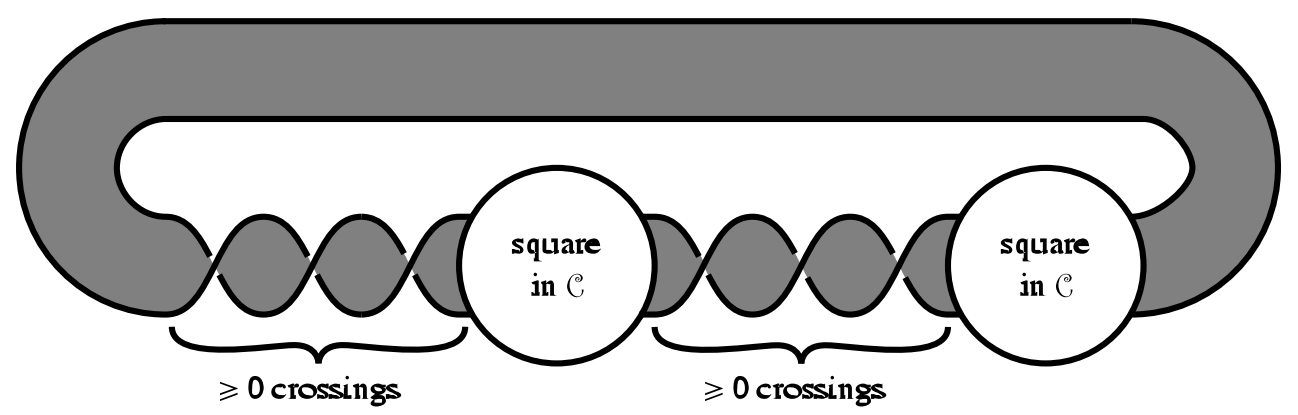

Figure 7.

Lemma 7. Let $S$ and $S^{\prime}$ be essential squares, isotoped in the complement of the crossings to minimise $\left|S \cap S^{\prime}\right|$. Then they intersect in zero or two points, and in the latter case, the two points of intersection lie in distinct regions of the diagram with the same colour.

Proof. The four sides of $S$ run through distinct regions of the diagram, as do the four sides of $S^{\prime}$. A side of $S$ intersects a side of $S^{\prime}$ at most once. So $\left|S \cap S^{\prime}\right|$ is at most four. However, if it is exactly four, then $S$ and $S^{\prime}$ run through the same regions, hence are isotopic and so can be ambient isotoped off each other. So, $\left|S \cap S^{\prime}\right|$ is at most three. It is even and therefore either zero or two. In the latter case, the regions containing these two points cannot have opposite colour, as each arc of $S-S^{\prime}$ and $S^{\prime}-S$ would then intersect the link projection an odd number of times. So one of the four complementary regions of $S \cup S^{\prime}$ would have only two 
points of intersection with the link projection in its boundary. As the diagram is prime, it would contain a single arc of the link projection and no crossings, and hence $S$ and $S^{\prime}$ could be ambient isotoped off each other.

Lemma 8. Let $R$ be a product complementary region of a non-empty collection $\mathcal{C}$ of disjoint essential squares. Then any essential square in $R$ that is characteristic must be parallel to square in $\mathcal{C}$.

Proof. Suppose that the white regions in $R$ form the horizontal boundary of $R$. Pick one such region $E$. Then (isotopy classes of) essential squares in $R$ are in one-one correspondence with (isotopy classes of) properly embedded arcs in $E$ with endpoints in distinct black regions. Let $S$ be an essential square in $R$ that is not parallel to any curve in $\mathcal{C}$ and that does not enclose a single crossing. Let $\alpha$ be the corresponding arc in $E$. Then each component of $\partial E-\partial \alpha$ contains an arc of intersection with a black region that is disjoint from $\partial \alpha$. Pick an $\operatorname{arc} \beta$ in $E$ joining these black regions. This corresponds to an essential square that cannot be isotoped off $S$. Hence, $S$ is not characteristic.

Lemma 9. Let $\mathcal{C}$ be a collection of disjoint non-parallel essential squares, such that

1. any essential square in the complement of $\mathcal{C}$ lies in a product complementary region, or is parallel to a square of $\mathcal{C}$, or encloses a single crossing;

2. if two product complementary regions of $\mathcal{C}$ are adjacent, they have incompatible product structures;

3. no square of $\mathcal{C}$ encloses a single crossing.

Then $\mathcal{C}$ is characteristic.

Proof. We will first show that each square in $\mathcal{C}$ is characteristic. So, consider an essential square $S$ and isotope it to intersect $\mathcal{C}$ minimally. Suppose that $S$ is not disjoint from $\mathcal{C}$, intersecting some curve $S^{\prime}$ of $\mathcal{C}$. By Lemma $7, S^{\prime}$ must intersect $S$ twice, and these two points of intersection lie in different regions of the diagram with the same colour, white say. Since all the curves in $\mathcal{C}$ are disjoint, any other curve of $\mathcal{C}$ intersecting $S$ must do so in the white regions. Hence, two components of $S-\mathcal{C}$ are arcs intersecting the link projection twice. Consider one such arc $\alpha$, and let $S_{1}$ be the curve of $\mathcal{C}$ containing its endpoints. Let $\beta$ be the $\operatorname{arc}(\mathrm{s})$ of $S-\mathcal{C}$ 
adjacent to $\alpha$, and let $S_{2}$ be the component(s) of $\mathcal{C}$ touching $\beta$, one of which is $S_{1}$. Then $\mathcal{N}\left(S_{1} \cup \alpha\right)$ is a product region. By (1), each boundary curve either is parallel to a curve of $\mathcal{C}$, or lies in a product complementary region of $\mathcal{C}$, or encircles a single crossing. Hence, $\mathcal{N}\left(S_{1} \cup \alpha\right)$ lies in a product complementary region $R_{1}$ of $\mathcal{C}$ (apart from an annular strip running along $S_{1}$ ). Similarly, $\mathcal{N}\left(S_{2} \cup \beta\right)$ lies in a product complementary region $R_{2}$ of $\mathcal{C}$. They have compatible product structures, contradicting (2). Hence, each square in $\mathcal{C}$ is characteristic.

Now, any characteristic square can be isotoped off $\mathcal{C}$, and so, by (1), is either parallel to a curve in $\mathcal{C}$ or lies in a product complementary region. In the latter case, it must be parallel to a curve in $\mathcal{C}$, by Lemma 8 . So, $\mathcal{C}$ consists of all the characteristic squares.

Lemma 10. The characteristic collection satisfies (1), (2) and (3) of Lemma 9.

Proof. Pick a maximal collection of disjoint non-parallel essential squares. This satisfies (1). Remove all squares that encircle a single crossing. The resulting collection satisfies (1) and (3). Remove a square if the two complementary regions either side of it are product regions and their union is again a product region. The collection still satisfies (1) and (3). Repeat this process as far as possible. The resulting collection satisfies (1), (2) and (3), and hence is the characteristic collection by Lemma 9 .

Lemma 11. Let $\mathcal{C}$ be a collection of disjoint non-parallel essential squares that bound a product region $R$. Suppose also that $\mathcal{C}$ is not a single square enclosing a single crossing. Then $R$ extends to a product complementary region of the characteristic collection.

Proof. Extend $\mathcal{C}$ to maximal collection of disjoint non-parallel essential squares that are disjoint from the interior of $R$. Then, as in the proof of Lemma 10, reduce it to collection satisfying (1), (2) and (3). The resulting collection is characteristic by Lemma 9 and, by construction, $R$ extends to a product complementary region of the characteristic collection.

Corollary 12. Each twist with more than one crossing lies in a product complementary region of the characteristic collection.

Proof. Apply Lemma 11 to a curve encircling the twist. 
We are now in a position to prove Lemma 4.

Proof of Lemma 4. Let $\mathcal{C}$ be the characteristic collection of squares for the diagram $D$. Each complementary product region is of the form shown in Figure 7. If this contains at least two twists, then there is a sequence of flypes that amalgamates all these twists into one. Perform all these flypes for all product regions, giving a new diagram $D^{\prime}$. The squares $\mathcal{C}$ in $D$ give squares $\mathcal{C}^{\prime}$ in $D^{\prime}$. Note that a complementary region of $\mathcal{C}$ is a product region before flyping if and only if the corresponding region of $\mathcal{C}^{\prime}$ is a product after flyping. Adjacent product regions of $\mathcal{C}$ have incompatible product structures, by Lemma 10 , and so adjacent product regions of $\mathcal{C}^{\prime}$ have incompatible product structures. So, by Lemma $9, \mathcal{C}^{\prime}$ is characteristic. Note that, by construction, each product complementary region of $\mathcal{C}^{\prime}$ contains at most one twist.

We claim that $D^{\prime}$ is twist-reduced. If not, it has a decomposition as in Figure 3 , where neither of the tangles $U$ nor $V$ is a row of bigons as shown. The squares $\partial U$ and $\partial V$ are essential and cobound a product region. By Lemma 11, this is part of a product region of $\mathcal{C}^{\prime}$. This contains at most one twist, and therefore either $U$ or $V$ is a row of bigons, as shown in Figure 3. Therefore $D^{\prime}$ is twist-reduced.

We now have to prove that $t\left(D^{\prime}\right) \geq t(D) / 2+1$. Let $\mathcal{C}_{+}$be the characteristic collection $\mathcal{C}$ of $D$, together with a curve enclosing each crossing that is not part of a longer twist. By Corollary 12, each twist lies in a product complementary region of $\mathcal{C}_{+}$.

Define a graph $G$ having a vertex for each complementary region of $\mathcal{C}_{+}$. Two vertices are joined by an edge if and only if the corresponding regions are adjacent. Denote the vertex set by $V(G)$ and the subset of the vertices that arise from a product region with at least one twist by $T(G)$. For any vertex $v$ of $V(G)$, let $\lambda(v)$ be its valence. Note that the vertices of valence one correspond to innermost regions of the diagram, which are necessarily a single twist, and hence lie in $T(G)$. Note also that the valence of a vertex in $T(G)$ is at least the number of twists that the corresponding region of $D$ contains. Now, $G$ is a tree, and so, by Euler characteristic,

$$
\sum_{v \in V(G)}(\lambda(v)-2)=-2 .
$$


Therefore,

$$
\sum_{v \in T(G)}(\lambda(v)-2)=-2-\sum_{v \in V(G)-T(G)}(\lambda(v)-2) \leq-2 .
$$

So,

$t(D) \leq \sum_{v \in T(G)} \lambda(v)=\sum_{v \in T(G)}(\lambda(v)-2)+2|T(G)| \leq-2+2|T(G)|=-2+2 t\left(D^{\prime}\right)$.

Rearranging this gives that $t\left(D^{\prime}\right) \geq t(D) / 2+1$.

\section{The GUTS OF THE CHECKERBOARD SURFACES' EXTERIORS}

Let $D^{\prime}$ be a twist-reduced prime alternating diagram. Let $B$ be its black checkerboard surface and let $R$ be its white bigon regions. Then we may view $R$ as a collection of discs properly embedded in $M_{B}$. Each disc of $R$ intersects the parabolic locus $P=\partial M \cap M_{B}$ transversely twice. Hence, $\mathcal{N}(R \cup P)$ is an $I$-bundle $E$ embedded in $M_{B}$. It is part of the characteristic submanifold of $M_{B}$. So, the guts of $M_{B}$ is the guts of $\operatorname{cl}\left(M_{B}-E\right)$, where the latter is given parabolic locus $\operatorname{cl}\left(\partial E-\partial M_{B}\right)$.

We can identify $\operatorname{cl}\left(M_{B}-E\right)$ explicitly. If each twist of $D^{\prime}$ consisting of white bigons is removed and replaced with a single crossing, the result is an alternating diagram $\hat{D}$ (of a new link). Let $\hat{B}$ be its black checkerboard surface. Then $\operatorname{cl}\left(M_{B}-\right.$ $E)$ is homeomorphic to $S^{3}-\operatorname{int}(\mathcal{N}(\hat{B}))$, the homeomorphism taking parabolic locus to parabolic locus. Hence, it suffices to analyse $\hat{B}$ and $\hat{D}$. It is clear that $\hat{D}$ is prime, but it may not be twist-reduced. Nevertheless, it is black-twist-reduced, which means that the implication of Figure 3 holds whenever the regions of the figure adjacent to both $U$ and $V$ are black. For example, the diagram in Figure 8 is black-twist-reduced, but not reduced. It in fact arises from the diagram in Figure 6 by assigning a checkerboard colouring, and then removing the white bigons.

Theorem 13. Let $D$ be a black-twist-reduced prime alternating diagram, with no white bigon regions. Let $B$ be its black checkerboard surface. Then

$$
\chi\left(\operatorname{Guts}\left(M_{B}\right)\right)=\chi\left(M_{B}\right) .
$$




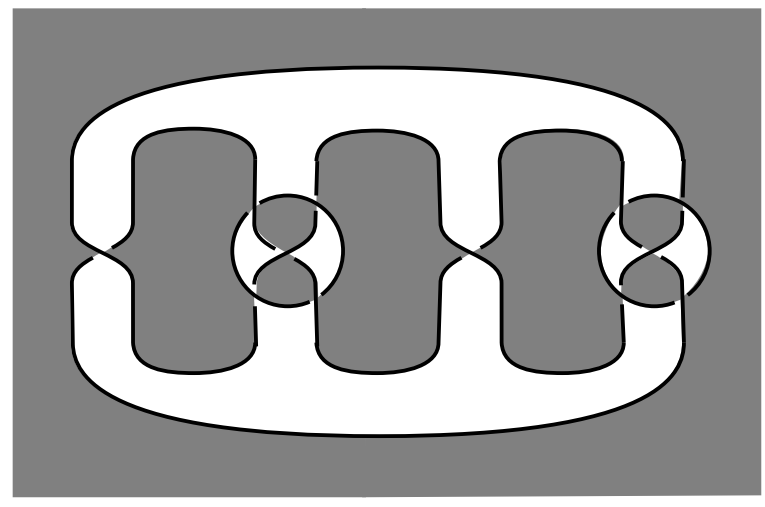

Figure 8.

Theorem 5 is an immediate corollary, since we may apply Theorem 13 to $\hat{D}$ and deduce that $\chi\left(\operatorname{Guts}\left(M_{B}\right)\right)=\chi\left(S^{3}-\operatorname{int}(\mathcal{N}(\hat{B}))\right.$. But $S^{3}-\operatorname{int}(\mathcal{N}(\hat{B}))$ is a regular neighbourhood of a graph, with two vertices and $r_{W}\left(D^{\prime}\right)$ edges. One vertex lies above the diagram, and one vertex lies below, and each edge runs between the vertices through a non-bigon white region of $D^{\prime}$. So its Euler characteristic is $2-r_{W}\left(D^{\prime}\right)$.

We now embark on the proof of Theorem 13. So, suppose that $D$ is a blacktwist-reduced prime alternating diagram with no white bigon regions. Let $A$ be a characteristic annulus for the characteristic submanifold of $M_{B}$. It is incompressible, and disjoint from the parabolic locus. It may be parabolically compressible, in which case it parabolically compresses to a product disc, which is a disc properly embedded in $M_{B}$ intersecting the parabolic locus in two transverse arcs. This product disc is essential in the sense that it is not boundary-parallel, provided $A$ is not boundary-parallel. We will prove the following result.

Theorem 14. Let $D$ be a connected black-twist-reduced prime alternating diagram with no white bigon regions. Let $B$ be its black checkerboard surface. Then

(i) $M_{B}$ contains no essential product discs, and

(ii) any incompressible parabolically-incompressible annulus $A$ that is disjoint from the parabolic locus separates off a Seifert fibred solid torus subset of the characteristic submanifold of $M_{B}$.

Proof. Suppose first that there is an essential product disc $S$. It is incompressible 
and parabolically incompressible, and so can be ambient isotoped into normal form. The intersection of $S$ with $W$ is a collection of arcs. Each of these arcs must run between distinct components of $S \cap \partial \mathcal{N}(B)$. For, otherwise, we may consider an arc of $S \cap W$ with endpoints in the same component of $S \cap \partial \mathcal{N}(B)$, that is outermost in $S$. This separates off a subdisc of $S$ that lies in a single ideal polyhedron and that has two points of intersection with the polyhedron's edges. Since the link diagram is prime, these must lie in the same edge, contradicting normality.

Suppose now that $S \cap W$ is non-empty. An outermost arc of $S \cap W$ in $S$ separates off a disc $S_{1}$. This lies in one of the ideal polyhedra of the link complement, and so gives a curve in the link diagram, as shown in Figure 9. Let $N$ be the region of the diagram containing $S_{1} \cap W$. Note that $S_{1} \cap N$ cannot separate off a single crossing in $\partial N$. For $D$ would then decompose as in Figure 3. However, the fact that $D$ is black-twist-reduced would imply that one of $U$ or $V$ in Figure 3 would contain a row of white bigons, whereas $D$ contains no white bigons.

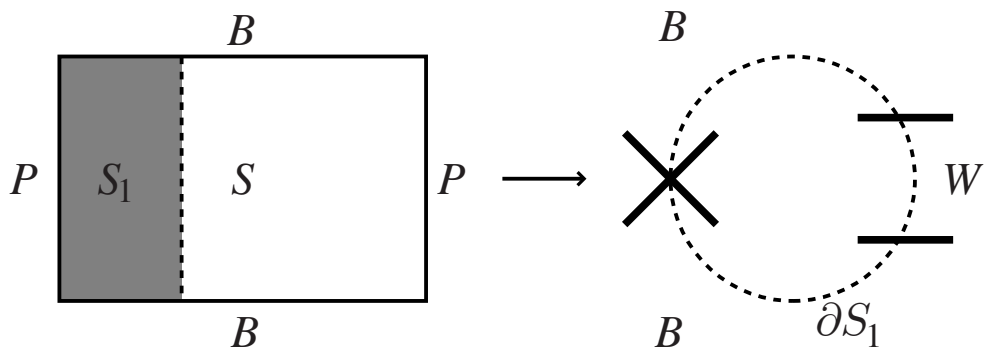

Figure 9.

The arc $S_{1} \cap W$ is part of a normal disc $S_{2}$ in the other ideal polyhedron. Both ideal polyhedra have the same boundary graph. So we may super-impose $\partial S_{1}$ and $\partial S_{2}$. Perform a small ambient isotopy so that they miss the crossings, ensuring that the new white side of $\partial S_{1}$ is disjoint from $\partial S_{2}$. The result is two essential squares. Perform a further isotopy in the complement of the crossings so that they have minimal intersection. Since $S_{1} \cap N$ cannot separate off a single crossing in $\partial N, \partial S_{1}$ and $\partial S_{2}$ must intersect in $N$. So, in the white regions, they have only a single point of intersection. (See Figure 10.) This contradicts Lemma 7. 


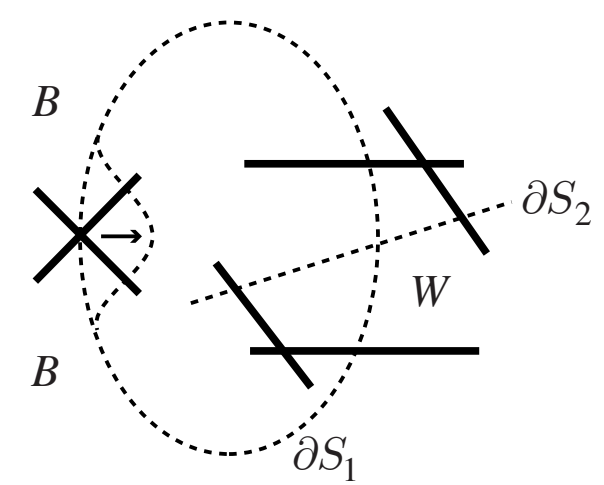

Figure 10.

Hence, the assumption that $S \cap W$ is non-empty is impossible. The disc $S$ therefore lies entirely above or below the diagram. It runs over two crossings. The fact that $D$ is black-twist-reduced gives that these two crossings are joined by a row of white bigons, again a contradiction. Thus, $M_{B}$ contains no essential product discs.

Now consider an incompressible parabolically-incompressible annulus $A$ that is disjoint from the parabolic locus. Again, we may place $A$ in normal form. As in the case of the product disc, the arcs $A \cap W$ must run between distinct boundary components of $A$.

Consider a disc $S_{2}$ lying between two adjacent $\operatorname{arcs} \alpha_{1}$ and $\alpha_{3}$ of $A \cap W$. Each is part of discs $S_{1}$ and $S_{3}$ properly embedded in the other ideal polyhedron. As before, super-impose $\partial S_{2}$ and $\partial S_{i}(i=1$ or 3$)$, and minimise their intersection with an ambient isotopy. If $R_{i}$ is the region of the diagram containing $\alpha_{i}$, then $\alpha_{i}$ cannot separate off a single crossing in $\partial R_{i}$. For this would imply the existence of a parabolic compression disc. See Figure 11. Hence, $\partial S_{2}$ and $\partial S_{i}$ intersect in the white region $R_{i}$. By Lemma 7, they have exactly one other point of intersection, in some other white region. Since $\partial S_{1}$ and $\partial S_{3}$ are disjoint, the only possibility for $\partial S_{1}, \partial S_{2}$ and $\partial S_{3}$ is as shown in Figure 12. 


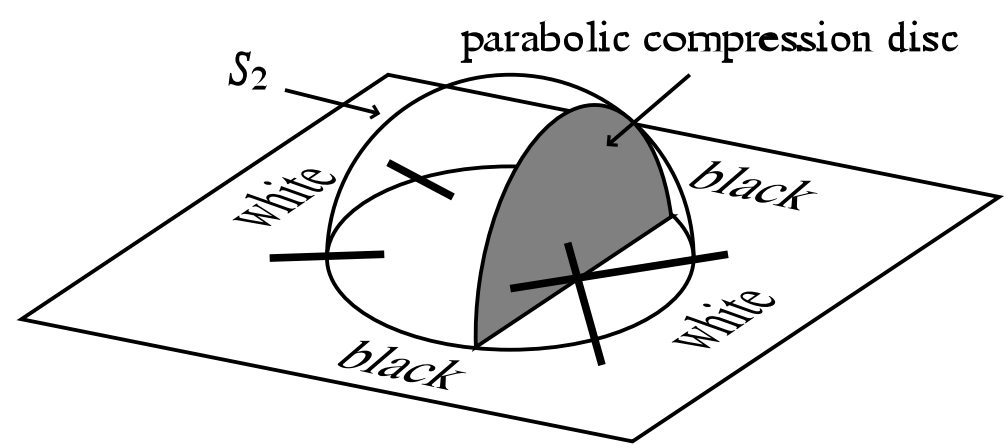

Figure 11.

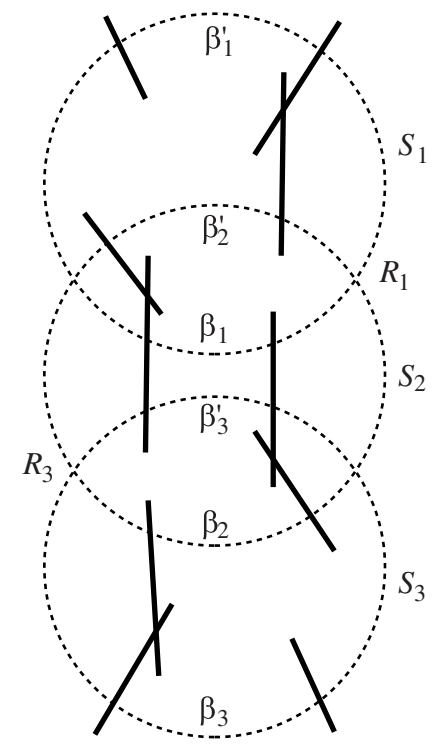

Figure 12 .

We term a disc subset of the diagram a unit if its boundary is an essential square, and its intersection with some white region of the diagram contains a single crossing. Note that a unit is uniquely specified by its two black sides, since these sides extend uniquely to a square and a square cannot bound units on both sides in a black-twist-reduced diagram with no white bigons. Two units are fused if

- they are disjoint;

- two of their black sides are parallel; and

- either the white boundary sides that separate off a crossing are not adjacent, or at least one of the units contains a single crossing.

Note that, if a unit is fused to some other unit, then either it contains a single 
crossing or its boundary is characteristic. For if it does not contain a single crossing, then its boundary has product regions with incompatible product structures on both sides. By Lemma 11, these extend to product complementary regions of the characteristic collection. So, the boundary of the unit is characteristic.

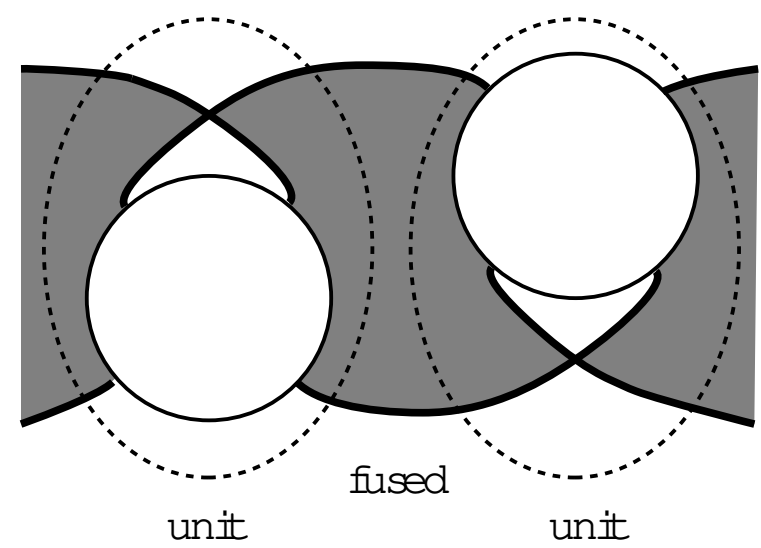

Figure 13.

Denote the $\operatorname{arcs} \partial A \cap S_{i}$ by $\beta_{i}$ and $\beta_{i}^{\prime}$, where $\beta_{1}, \beta_{2}$ and $\beta_{3}$ all lie in the same component of $\partial A$. We deduce from Figure 12 that $\beta_{1}$ and $\beta_{2}$ form opposite black edges of a unit, as do $\beta_{2}$ and $\beta_{3}$, and that these units are fused. This argument may be applied to the two squares $\left(S_{2}\right.$ and $\left.S_{4}\right)$ either side of $S_{3}$. If $\beta_{4}$ is the arc of $\partial A \cap S_{4}$ adjacent to $\beta_{3}$, we deduce that $\beta_{3}$ and $\beta_{4}$ form opposite black edges of a unit, which is fused to the unit bounded by $\beta_{2}$ and $\beta_{3}$. Continue in this way. The resulting units all have non-intersecting boundaries (up to isotopy) since they are characteristic or separate off a single crossing. They cannot be non-trivially nested, since they are fused to other units. Hence, the only possibility is that the units start to repeat. That is, they are all fused in a circular fashion, as in Figure 14.

We observe from Figure 12 that, for either of the polyhedra $P_{i}$, each curve of $A \cap \partial P_{i}$ encircles two units. These curves are all disjoint. Also, if two discs of $A \cap P_{i}$ have a single disc between them (for example, $S_{1}$ and $S_{3}$ ), then two of their black edges are parallel. There is therefore a constant number $(n$, say) of copies of each curve. Thus, $A$ is $n$ parallel copies of a surface. Since $A$ is connected, $n=1$. Therefore, the intersection of $A$ with the boundary of each ideal polyhedron is as shown in Figure 14. (There, the case $|A \cap W|=6$ is shown, but $|A \cap W|$ may be any even integer greater than three.) 
So, for each ideal polyhedron $P_{i}$, one component of $P_{i}-A$ is a ball with boundary a product region disjoint from the ideal vertices. The intersection of the product region with $W$ is two discs, one in the central region of Figure 14, one in the outer region. These two balls, one in each ideal polyhedron, glue together to form the required Seifert fibred solid torus.

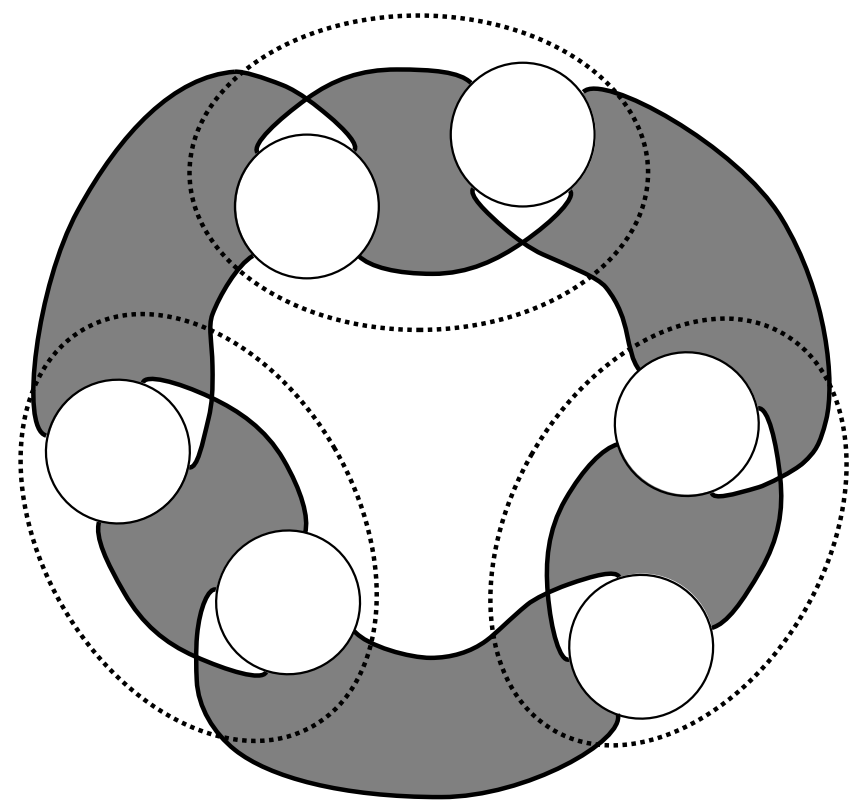

Figure 14 .

Proof of Theorem 13. Consider a bounding annulus of the characteristic submanifold of $M_{B}$. By Theorem 14, it is either boundary parallel or separates off a Seifert fibred solid torus. This Seifert fibred solid torus is part of the characteristic submanifold. We claim that the part of $M_{B}$ on the other side of this annulus cannot also be part of the characteristic submanifold. If it were, it would have to be an $I$-bundle. If this intersected the parabolic locus, it would contain an essential product disc, contradicting Theorem 14. Its bounding annuli must each separate off a Seifert fibred solid torus by Theorem 14. But then $M_{B}$ contains no parabolic locus, which is a contradiction, proving the claim. So, the characteristic submanifold of $M_{B}$ is a collection of Seifert fibred solid tori attached to the guts of $M_{B}$ via annuli. Since each solid torus and each annulus has zero Euler characteristic, $\chi\left(\operatorname{Guts}\left(M_{B}\right)\right)=\chi\left(M_{B}\right)$. 


\section{Application: COnVERGent SEQuences}

\section{OF ALTERNATING LINK COMPLEMENTS}

As a sample application of the main theorem of this paper, we show that the only possible limits of sequences of hyperbolic alternating link complements converging in the geometric topology are the 'obvious' ones.

Corollary 2. A complete finite volume hyperbolic 3-manifold is the limit of a sequence of distinct hyperbolic alternating link complements if and only if it is a hyperbolic augmented alternating link complement.

Proof. It is well known that a sequence of complete finite volume hyperbolic 3manifolds $M_{i}$ converges in the geometric topology to a finite volume hyperbolic 3-manifold $M_{\infty}$ if and only if all $M_{i}$ sufficiently far along this sequence are obtained by Dehn filling $M_{\infty}$, so that none of the surgery slopes have a constant subsequence. Hence, one way of obtaining a convergent sequence of alternating hyperbolic link complements is to start with a single augmented alternating link and perform surgeries along the augmenting unknots, where the surgery coefficients are chosen so that the resulting diagrams are alternating. Thus, any hyperbolic augmented alternating link complement is certainly the limit of a sequence of distinct hyperbolic alternating link complements. We must show that the converse also holds.

Consider a collection of distinct hyperbolic alternating knots $\left\{K_{i}\right\}$ with complements that converge in the geometric topology to a finite volume hyperbolic 3 -manifold. Their volumes are bounded. Therefore, by the main theorem of this paper, the twist numbers of their alternating diagrams are bounded. In $\S 2$, we showed how a link with a diagram $D$ is obtained by surgery along crossing circles in a link with a diagram having at most $6 t(D)$ crossings. When the original diagram is alternating, the new diagram is augmented alternating. Since the twist numbers of the $K_{i}$ are bounded, so are the crossing numbers of these augmented alternating links $L_{i}$. Thus, there are only finitely many possible $L_{i}$. We may therefore pass to a subsequence in which $L_{i}$ is a constant link. The $K_{i}$ in this subsequence have alternating diagrams obtained by surgically replacing each crossing circle tangle with a twist. Pass to a subsequence so that, at every twist, the number of crossings is either constant or tends to infinity. Then, the links in this subsequence are 
all obtained by Dehn filling a single augmented alternating link $L$, so that none of the surgery coefficients have a constant subsequence. So, $L$ is the limit of the sequence.

Corollary 3. The set of all hyperbolic alternating and augmented alternating link complements is a closed subset of the set of all complete finite volume hyperbolic 3-manifolds, in the geometric topology.

Proof. Consider a convergent sequence of hyperbolic alternating and augmented alternating link complements. We wish to show that the limit hyperbolic manifold is an alternating or augmented alternating link complement. Arbitrarily close to each augmented alternating link in the geometric topology, there is an alternating link. Hence, we may assume that the sequence consists only of alternating links. If it has a constant subsequence, the limit is an alternating link complement. If it has no constant subsequence, the limit is an augmented alternating link complement, by Corollary 2 .

Mathematical Institute, Oxford University, 24-29 St Giles', Oxford OX1 3LB, England. 


\section{APPENDIX}

\section{IAn Agol and Dylan Thurston}

In this appendix, we improve on Lackenby's upper bound on the volume of links in terms of the twist number of the projection diagram, proven in section 2 of the paper. We will use the same notation as in section 2 .

Theorem. Given a projection diagram $D$ of a link $K$ with twist number $t(D)$, $\operatorname{Vol}\left(S^{3}-K\right) \leq 10 v_{3}(t(D)-1)$. Moreover, there is a sequence of links $K_{i}$ such that $\operatorname{Vol}\left(S^{3}-K_{i}\right) / t\left(D_{i}\right) \rightarrow 10 v_{3}$.

Proof. We use Lackenby's approach, by taking the link $K$ and creating an augmented alternating link $L$ which has components lying flat in the projection plane which are bound together by crossing circles.

We will describe two decompositions of $S^{3}-L$, in order to get an upper bound on the volume. The first decomposition is obtained by taking the planar surface lying in the projection plane, and taking the 2-punctured disks bounding each crossing loop. The 2-punctured disks are totally geodesic in the complement of $L$ [2], and there is a reflection through the projection plane exchanging the polyhedra, and preserving the planar surface in the projection plane. So this surface is totally geodesic, and the 2-punctured disks are perpendicular to the planar surface. These surfaces determine a decomposition of $S^{3}-L$ into two ideal polyhedra $P_{1}$ and $P_{2}$ with their faces identified in pairs (see Figure 15). These polyhedra $P_{1}$ and $P_{2}$ are identical, and have the property that the faces may be checkerboard colored dark and white so that the dark faces are triangles which come in pairs sharing a vertex each, like a bow-tie. $S^{3}-L$ is obtained by folding the bowties in $P_{1}$ along each vertex to glue the pairs of triangles together, then doubling along the rest of the faces. 


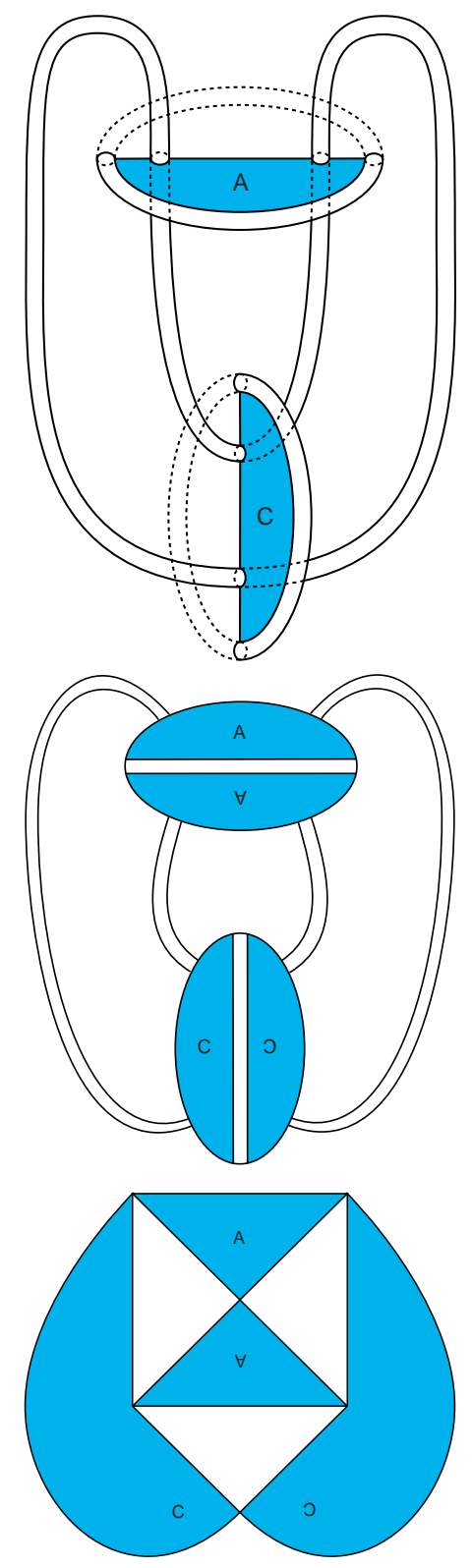

Figure 15: Decomposing the complement of $L$ into ideal polyhedra

The second decomposition is into tetrahedra. This is obtained by putting vertices $v_{1}$ and $v_{2}$ in the interior of $P_{1}$ and $P_{2}$, and coning the vertices to the faces of the polyhedra. Each dark face of $P_{1}$ and $P_{2}$ gets coned off to two tetrahedra, and each white face gets coned off to two pyramids. For each white face, we do a stellar subdivision on the two pyramids containing it. That is, we remove the face, and add an edge dual to the face connecting $v_{1}$ and $v_{2}$. Then we add in triangles 
around the new edge to divide the region into tetrahedra. If the face has $d$ edges, then this divides the two cones into $d$ tetrahedra.

To compute the total number of tetrahedra in this triangulation, notice that each crossing loop contributes 6 edges to $P_{1}$ and $P_{2}$. Thus, the total number of edges in the white faces will be $6 t(D)$, which contributes $6 t(D)$ tetrahedra. Each crossing loop also contributes 2 dark triangles each to $P_{1}$ and $P_{2}$, giving 4 tetrahedra when we cone off to $v_{1}$ and $v_{2}$. Thus, we have a total of at most $10 t(D)$ tetrahedra in this decomposition.

We may reduce the number of tetrahedra by choosing an ideal vertex, and collapsing the edges adjoining $v_{1}$ and $v_{2}$ to this vertex. We then simplify the resulting cell decomposition, by collapsing monogons to vertices, bigons to single edges, and parallel triangles to single triangles, to get an ideal triangulation. The vertex we collapse to is adjacent to two dark faces and two white faces (in the polyhedral decomposition into $P_{1}$ and $P_{2}$ ). Thus, when we collapse the vertices $v_{1}$ and $v_{2}$ to an ideal point, we collapse the 4 tetrahedra adjacent to the dark faces to triangles. All the white faces have degree $\geq 3$, since we have assumed that no two crossing loops are parallel. So we also collapse $\geq 6$ tetrahedra going through the two white faces to triangles. Thus, we may find an ideal triangulation with at most $10 t(D)-10$ tetrahedra.

This allows us to bound the volume of $S^{3}-L$, as in section 2, by straightening the triangulation, and bounding the volume of each simplex by $v_{3}$, to conclude that $\operatorname{Vol}\left(S^{3}-L\right) \leq 10 v_{3}(t(D)-1)$.

Now, we consider the second claim of the theorem, which shows that we have obtained the optimal constant. As motivation, we will consider the infinite augmented link $C$ in $\mathbb{R}^{3}$ resembling a chain link fence, which realizes exactly the upper bound on volume density of $10 v_{3}$ per crossing loop. 


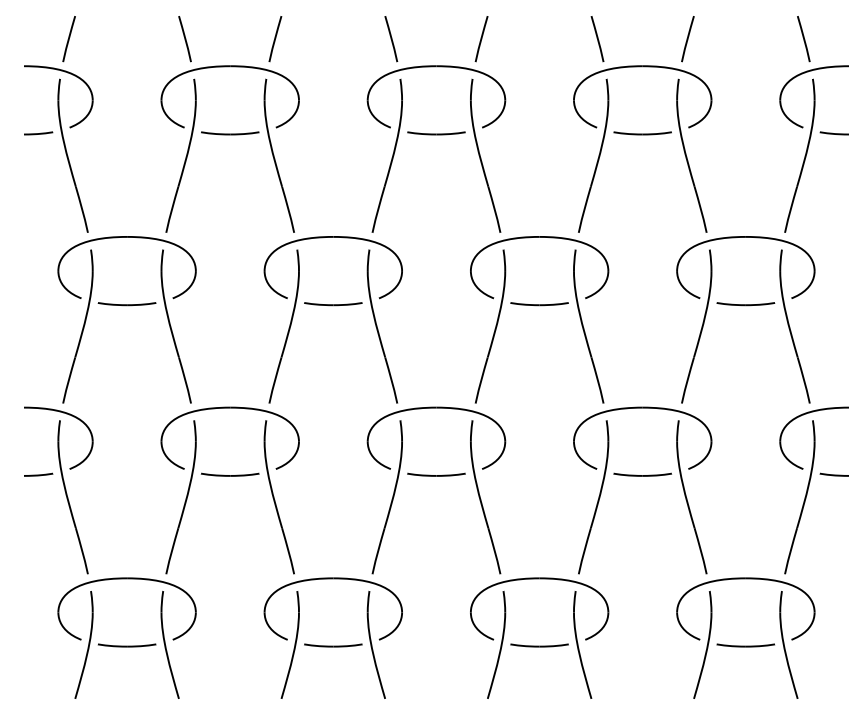

Figure 16: The chain fence link

Using the decomposition of the complement of $C$ into $P_{1}$ and $P_{2}$, we get an infinite polyhedron with faces alternating between triangles and hexagons, like a union of stars of David (see Figure 17).

This polyhedron has a natural realization as a right angled polyhedron, by taking the tessellation with regular triangles and hexagons, and putting a circle around each face. This is the same as taking the regular hexagonal packing of circles, and putting a circle around each interstitial region (see Figure 17). So all the circles are either disjoint, tangent, or orthogonal. Each circle bounds a geodesic plane in the upper half space model of $\mathbb{H}^{3}$, together cutting out a rightangled polyhedron. Thus, we get a complete hyperbolic structure on the link complement when we glue the polyhedra together to get $\mathbb{R}^{3}-C$. 


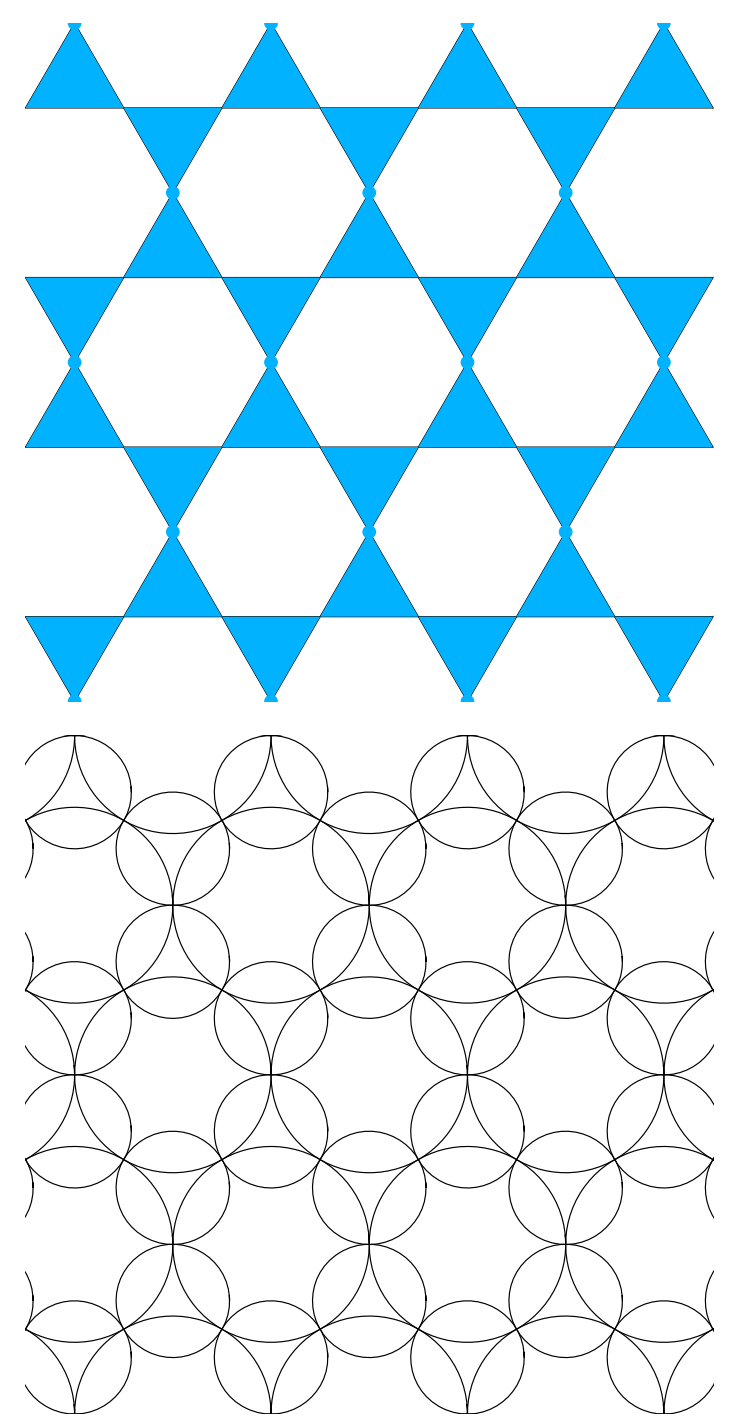

Figure 17: The infinite polyhedron

Remark. There are actually many infinite augmented alternating link complements with the same volume density. Take any decomposition of the triangles in the star of David tessellation (Figure 17) into bow-ties. Then folding the bowties to glue the triangles together and doubling gives an ideal cell structure of an infinite augmented alternating link complement with volume density $10 v_{3}$ per crossing circle.

We may form a sequence of augmented links $C_{i}$ by taking the 2222-orbifold quotient of the link $C$, for an increasing sequence of fundamental domains for the 
2222-orbifold, and deleting the orbifold cone axes. The polyhedra of the links $C_{i}$ are obtained by taking the 2222-orbifold quotient of the star of David tessellation, and deleting the orbifold cone points. That these polyhedra are realized by rightangled polyhedra in $\mathbb{H}^{3}$ is a consequence of Andreev's theorem, see for example [8]. Coning the points to a vertex, we get a division of this polyhedron into tetrahedra and cones on hexagons. Taking the circle packing corresponding to the white faces of the polyhedron, we get a circle packing on $S^{2}$ such that all but four circles are adjacent to exactly 6 other circles. Moreover, as $i \rightarrow \infty$, the majority of the circles will have a packing around them which is combinatorially equivalent to $n$ generations of the regular hexagonal circle packing, where we may assume that $n \rightarrow \infty$ as $i \rightarrow \infty$. Sending the cone vertex to $\infty$ in the upper half space model of $\mathbb{H}^{3}$, we see a circle surrounded by $n$ generations of the regular hexagonal packing. A theorem of Rodin and Sullivan [7] then shows that as $n \rightarrow \infty$, the ratios of radii of the circles adjacent to the central circle go to 1 . Thus, the majority of faces coned to $\infty$ then approaches either a regular tetrahedron, or a cone on a regular ideal hexagon. So as $i \rightarrow \infty$, the density of volume per crossing circle of $S^{3}-C_{i}$ approaches that of $\mathbb{R}^{3}-C$, which is $10 v_{3}$. Then we may form links $K_{i}$ by taking $\pm 1 / q$ surgeries on the links $C_{i}$ to get alternating links, where we choose $q \rightarrow \infty$ fast enough that $\operatorname{Vol}\left(K_{i}\right) / \operatorname{Vol}\left(C_{i}\right) \rightarrow 1$, which we may do by Thurston's hyperbolic Dehn surgery theorem [8].

\section{REFERENCES}

1. C. Adams, Thrice-punctured spheres in hyperbolic 3-manifolds, Trans. Am. Math. Soc. 287 (1985) 645-656.

2. C. Adams, Augmented alternating link complements are hyperbolic, Lowdimensional Topology and Kleinian groups, London Math. Soc. Lecture Note Series. 112, Cambridge Univ. Press (1986)

3. I. Agol, Lower bounds on volumes of hyperbolic Haken 3-manifolds, Preprint (1999)

4. R. Benedetti And C. Petronio, Lectures on Hyperbolic Geometry, Springer-Verlag (1992). 
5. W. Menasco, Closed incompressible surfaces in alternating knot and link complements, Topology 23 (1984) 37-44.

6. W. Menasco and M. Thistlethwaite, The classification of alternating links., Ann. Math. 138 (1993) 113-171.

7. B. Rodin and D. Sullivan, The convergence of circle packings to the Riemann mapping. J. Differential Geom. 26 (1987) 349-360.

8. W. Thurston, The geometry and topology of three-manifolds, Lecture notes from Princeton University (1978-80).

Department of Mathematics and Statistics, University of Melbourne, Parkville, VIC 30120, Australia.

Department of Mathematics, Harvard University,

1 Oxford Street, Cambridge, MA 02138, USA. 